\title{
Hydrodynamic Pair Attractions Between Driven Colloidal Particles
}

\author{
Yulia Sokolov, ${ }^{1}$ Derek Frydel, ${ }^{1}$ David G. Grier, ${ }^{2}$ Haim Diamant,${ }^{1}$ and Yael Roichman ${ }^{1, *}$ \\ ${ }^{1}$ Raymond and Beverly Sackler School of Chemistry, Tel Aviv University, Tel Aviv 69978, Israel \\ ${ }^{2}$ Department of Physics and Center for Soft Matter Research, New York University, New York, NY 10003
}

(Dated: June 15, 2018)

\begin{abstract}
Colloidal spheres driven through water along a circular path by an optical ring trap display unexpected dynamical correlations. We use Stokesian Dynamics simulations and a simple analytical model to demonstrate that the path's curvature breaks the symmetry of the two-body hydrodynamic interaction, resulting in particle pairing. The influence of this effective nonequilibrium attraction diminishes as either the temperature or the stiffness of the radial confinement increases. We find a well defined set of dynamically paired states whose stability relies on hydrodynamic coupling in curving trajectories.
\end{abstract}

PACS numbers: 82.70.Dd, 87.80.Cc

Position correlations among particles in thermal equilibrium stem solely from their potential energy. Although the motions of fluid-suspended particles are correlated via flows that they induce (hydrodynamic interactions), this has no effect on the equilibrium configurations. For particles driven out of equilibrium, however, this is no longer the case, and they may get closer or drift apart in the absence of an interaction potential. Examples are found in studies of nonequilibrium particle aggregation and droplet coalescence under flow (e.g., [1]), aggregation of blood cells in channel flow 2], and strong correlations in sedimenting suspensions [3]. Nonequilibrium pair attractions have been reported in simulations of particles, embedded in a flowing bath of smaller particles [4], or driven through such a bath [5]. Yet, all these phenomena are observed in the presence of either many-body effects or nonuniform flow, where spatially separated particles experience a different drive.

It is not self-evident that two identical particles, having no intrinsic interactions, can attract or repel each other under conditions of identical driving [6]. This is because a uniaxial driving force, breaking rotational symmetry, is generally insufficient to break the symmetry of the hydrodynamic interaction on the two-body level. A familiar example is an isolated pair of noninteracting colloidal particles, sedimenting under gravity in an unbounded viscous fluid. One particle drags the other to the exact same extent that the other pushes it; hence, their separation remains constant. One way to break this symmetry is to introduce a boundary. Thus, those two sedimenting particles, when falling away from a horizontal wall, experience a hydrodynamic force that draws them together 6]. Another way is to consider more than two particles. For example, driving a one-dimensional array of particles by a uniform flow through a slit creates hydrodynamic restoring forces, resulting in density waves [7].

In this Letter we show that the symmetry of the hydrodynamic interactions of identically driven particles in a fluid can be spontaneously broken on the two-body level if their trajectories curve. The result is the formation of dynamically paired states, whose stability determines the steady transport properties of the system.

Our samples consist of aqueous dispersions of colloidal polystyrene particles, sealed in a $50 \mu \mathrm{m}$ thick gap between a glass microscope slide and a coverslip. The Debye-Hückel screening length is of order $10 \mathrm{~nm}$, implying that the charge-stabilized spheres' electrostatic interactions are negligible at micrometer-scale separations. Individual spheres are captured and driven through water by extended optical traps, created with the holographic optical trapping technique [8, 9]. In our implementation [10], we use a helical mode of light, which focuses to a ring-like optical trap known as an optical vortex 8, 10, 11]. Particles are drawn to the optical vortex by forces directed by intensity gradients [12], and are propelled around it by radiation pressure arising from the helical beam's phase gradients [13]. Adaptive optics methods are employed to reduce variations in the tangential driving force [9, 14]. The optical force profile, as inferred from the motion of a single particle, is shown in Fig. 1(a), revealing a roughness of about $30 \%$. The optical vortex is powered with $2.5 \mathrm{~W}$ of laser light at a vacuum wavelength of $532 \mathrm{~nm}$, which suffices to drive trapped particles with tangential velocities $V_{\theta}$ of a few tens of micrometers per second, corresponding to angular velocities $\Omega$ of a few radians per second. Standard methods of digital video microscopy [15] are used to track the particles at a rate of 30 frames/s with $20 \mathrm{~nm}$ accuracy.

We present results for two sets of experimental parameters (particle diameter $\sigma$, vortex radius $R$, tangential driving force $F_{\theta}$, and radial trapping stiffness $k_{r}$ ), as listed in Table \. Experiments were performed for numbers of particles ranging from $N=1$ to $N=12$, running around the ring in single-file trajectories. The particles' inertia may be ignored because of the negligible Reynolds number, $\operatorname{Re}=\rho V_{\theta} \sigma / \eta \sim 10^{-5}$, where $\eta$ and $\rho$ are the viscosity and mass density of water, respectively. Random thermal forces contribute fluctuations to the circulating particles' velocities [16]. The strength of the driving relative to thermal fluctuations is characterized by the Péclet 

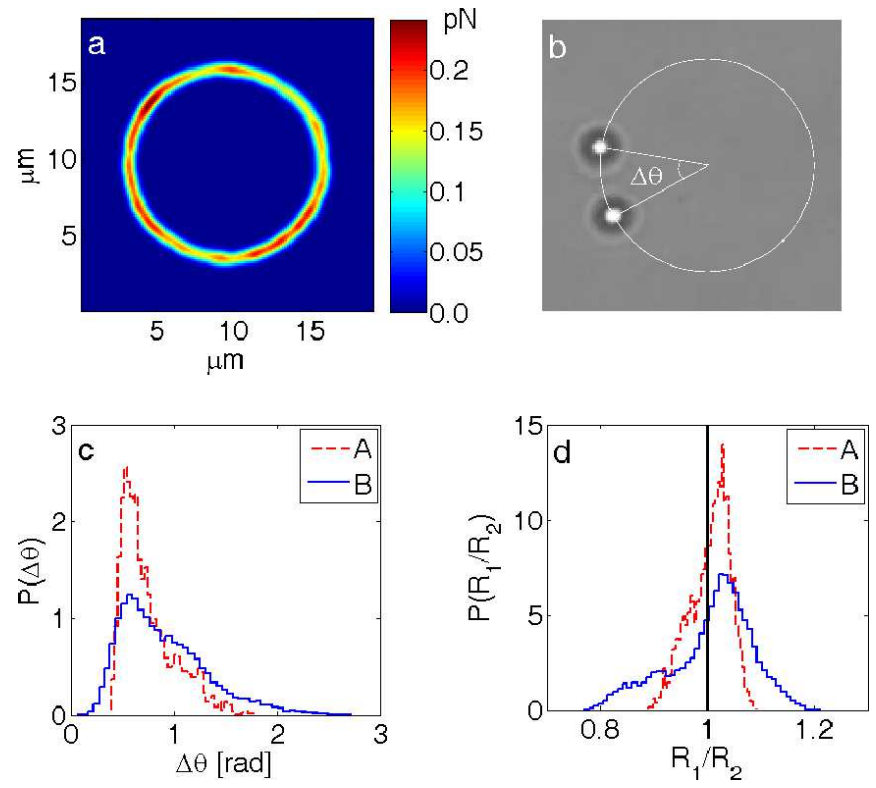

FIG. 1: (color online) (a) Magnitude of the tangential component of the force exerted by an optical vortex (system A) on a single colloidal sphere. (b) Visualization of a pair having an angular separation $\Delta \theta$. (c,d) Distributions of experimentally measured angular separation (c) and ratio between the radii of the leading and trailing particles (d) in a two-particle vortex for the two systems.

number, $\mathrm{Pe}=F_{\theta} \sigma /\left(k_{\mathrm{B}} T\right) \simeq 90$ and 74 , respectively, for systems $\mathrm{A}$ and $\mathrm{B}$, where $k_{\mathrm{B}}$ is Boltzmann's constant, and $T$ the absolute temperature. The ratio of the radial confinement strength and tangential driving, $\kappa=k_{r} R / F_{\theta}$, serves as another characteristic parameter. We estimate $\kappa \simeq 30$ for both systems.

Because the particles have no intrinsic long-range interactions and because they are driven by the same optical force, a pair of particles might be expected to diffuse independently and thus sample all possible separations with uniform probability. Experimentally, however, the two particles enter into a long-lived paired state [17], as quantified in the histograms of angular separations, Fig. 1(c). The narrower distribution observed for system $\mathrm{A}$ is consistent with its larger Pe.

The observed correlation between the particles' mo-

\begin{tabular}{ccccc}
\hline \hline system & $\sigma(\mu \mathrm{m})$ & $R(\mu \mathrm{m})$ & $F_{\theta}(\mathrm{pN})$ & $k_{r}(\mathrm{pN} / \mu \mathrm{m})$ \\
\hline $\mathrm{A}$ & $1.48 \pm 0.08$ & $6.25 \pm 0.05$ & $0.25 \pm 0.08$ & $1.2 \pm 0.3$ \\
$\mathrm{~B}$ & $0.97 \pm 0.03$ & $6.19 \pm 0.05$ & $0.31 \pm 0.09$ & $1.7 \pm 0.8$ \\
\hline \hline
\end{tabular}

TABLE I: The two experimental parameter sets: particle diameter $\sigma$, vortex radius $R$, tangential driving force $F_{\theta}$, radial confinement stiffness $k_{r}$. We calculate $F_{\theta}$ from the mean tangential velocity in a single-particle vortex, $F_{\theta}=V_{\theta} / B_{\mathrm{s}}$, and $k_{r}$ from the single particle's mean-square radial fluctuations assuming thermal equipartition [16], $k_{r}=k_{\mathrm{B}} T /\left\langle(\Delta r)^{2}\right\rangle$. tions must arise from the interplay of optical and hydrodynamic forces acting on them. A single sphere circulates around a smooth optical vortex with a uniform tangential velocity, $V_{\theta}=B_{\mathrm{s}} F_{\theta}$, where $B_{\mathrm{s}}=(3 \pi \eta \sigma)^{-1}$ is the sphere's Stokes self-mobility. As a sphere moves, it creates a flow field $\mathbf{G}(\mathbf{r}) \mathbf{F}_{\theta}$ at position $\mathbf{r}$. A second sphere placed on the optical vortex at $\mathbf{r}$ experiences not only the optical force $F_{\theta}$, but also the drag force due to its neighbor's flow. To leading order in $\sigma / r$, the change in the tangential velocity of the second particle due to its neighbor is simply the local tangential flow velocity, and $\mathbf{G}$ is given by the Oseen tensor [18], $G_{i j}(\mathbf{r})=(8 \pi \eta r)^{-1}\left(\delta_{i j}+r_{j} r_{j} / r^{2}\right)$, $i, j=x, y, z$. Applying this result to our case of two spheres on a ring of radius $R$, separated by an angle $\theta_{i j}=\theta_{j}-\theta_{i}$, we get for the tangential velocity,

$$
\begin{aligned}
V_{\theta}\left(\theta_{i j}\right) & \simeq\left[B_{\mathrm{s}}+g\left(\theta_{i j}\right)\right] F_{\theta}, \quad \text { where } \\
g\left(\theta_{i j}\right) & =\frac{1+3 \cos \theta_{i j}}{16 \pi \eta R \sqrt{2\left(1-\cos \theta_{i j}\right)}} .
\end{aligned}
$$

Equation (11) is symmetric under particle exchange, $\theta_{i j} \rightarrow$ $\theta_{j i}=-\theta_{i j}$, implying that the tangential component of the hydrodynamic coupling cannot account for the observed pairing. Because the circulating particles follow a curved path, however, the flow field due to particle $i$ generally has a component normal to the trajectory at the position of particle $j$. (See [17] for a theoretical visualization of the flow field.) Since the particle is held radially by the trap, this normal flow component gives rise to a radial force,

$$
\begin{aligned}
F_{r}\left(\theta_{i j}\right) & \simeq B_{\mathrm{s}}^{-1} h\left(\theta_{i j}\right) F_{\theta}, \quad \text { where } \\
h\left(\theta_{i j}\right) & =\frac{3 \sin \theta_{i j}}{16 \pi \eta R \sqrt{2\left(1-\cos \theta_{i j}\right)}} .
\end{aligned}
$$

This hydrodynamic force displaces the particle radially until it is counter-balanced by an equal optical force.

The fact that $h\left(\theta_{i j}\right)$ is antisymmetric under particle exchange implies that the two particles are displaced $o p$ positely in the radial direction. As is depicted schematically in Fig. 2 the leading particle is shifted to a slightly larger radius, $R_{1}>R$, and the trailing particle to a slightly smaller one, $R_{2}<R$. This spontaneous symmetry breaking is observed in the statistics of measured trajectories, plotted in Fig. I(d). If the tangential force $F_{\theta}$ is assumed constant, the radial displacement increases the trailing particle's angular velocity relative to that of the leading particle, and so causes the particles to form a hydrodynamically bound pair.

Pairing also appears in the dynamics of systems comprising more than two spheres. Driven rings of hydrodynamically coupled spheres thus constitute an interesting model system for studying spontaneous symmetry breaking in many-body systems out of equilibrium. Figure 3(a) shows the measured collective mobility as a function of particle number $N$. The increase in the circulation rate 


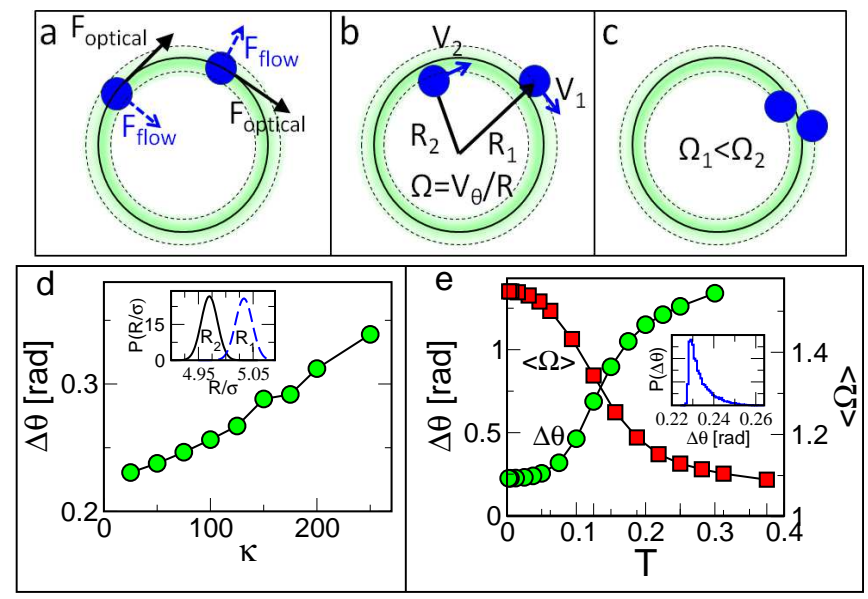

FIG. 2: (color online). ( $\mathrm{a}-\mathrm{c})$ The pairing mechanism. As optical forces drive the particles along the ring, the resulting fluid flow pushes the leading particle outward and pulls the trailing one inward. Once displaced radially, the particles' angular velocities change, making the trailing particle catch up with the leading one. (d,e) Simulation results for the effect of radial confinement strength (d) and temperature (e) on the average angular separation (green circles) and average angular velocity (red squares) in a two-particle vortex. The insets show the distributions of radial positions of the leading $\left(R_{1}\right)$ and trailing $\left(R_{2}\right)$ particles, and the pair's angular separation, for $T=0.0375$ and $\kappa=25$.

due to a decrease in the hydrodynamic drag is more pronounced for even values of $N$. In systems with odd $N$ the tendency to form pairs leaves one particle unpaired, which reduces the influence on the collective drag.

Let us focus on the case of even $N$ and consider an idealized configuration of $N / 2$ pairs, evenly positioned along the ring. In the absence of hydrodynamic interactions, each pair will move with tangential velocity $V_{\theta}=B_{\mathrm{p}} F_{\theta}$, where $B_{\mathrm{p}}=\alpha B_{\mathrm{s}}$ is the mobility of a pair ( $\alpha$ being a prefactor of order 1). Including tangential hydrodynamic couplings as above, we get for the collective mobility,

$$
\frac{B_{\mathrm{col}}}{B_{\mathrm{s}}}=\frac{V_{\theta}(N)}{V_{\theta}(1)}=\alpha+2 B_{\mathrm{s}}^{-1} \sum_{n=1}^{N / 2-1} g(4 \pi n / N),
$$

where $g(\theta)$ has been defined in Eq. (11). In Fig. 3(a) we compare the experimentally measured collective mobility, for even $N$, with the one predicted by Eq. (3), using the vertical shift $\alpha$ as a fitting parameter. Despite the simplifying assumptions underlying Eq. (3), a qualitative correspondence is obtained for $\alpha=1.10 \pm 0.02$. We attribute the larger discrepancies for $N=2$ and $N=4$ to larger deviations from the idealized configuration of fixed pairs in these dilute systems.

We systematically investigated how the pairing dynamics depend on Pe and $\kappa$ by performing Stokesian Dynamics simulations [19]. The simulated particles are restricted to the plane and confined to a ring of radius $R=5 \sigma$ by a harmonic radial potential of stiffness $k_{r}$. A
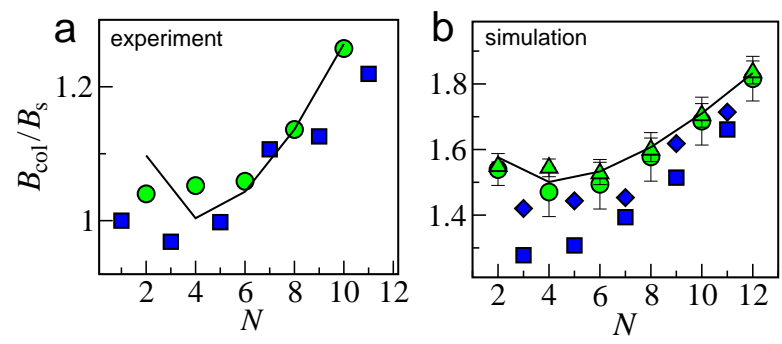

FIG. 3: (color online). Collective mobility, scaled by the single-particle mobility, as a function of particle number. (a) Experimental results for even $N$ (green circles) and odd $N$ (blue squares), measured from the ratio of mean velocities for $N$ particles and for a single particle in system A. (b) Simulation results for even $N$ (green circles, $T=0.1$; green triangles $T=0.02$ ) and odd $N$ (blue squares, $T=0.1$; blue diamonds, $T=0.02)$, at $\kappa=25$. The solid lines are theoretical fits for even $N$ [Eq. (3)] with $\alpha=1.10 \pm 0.02$ (experiment) and $\alpha=1.57 \pm 0.05$ (simulation). Error bars are smaller than symbols unless otherwise indicated.

constant tangential force $F_{\theta}$ drives the particles around the ring, and a repulsive Weeks-Chandler-Anderson pair interaction [20] accounts for collisions. Hydrodynamic interactions between particles are modeled using the manybody Rotne-Prager mobility tensor [21, 22]. Temperature is introduced via a Gaussian-distributed random force that obeys the fluctuation-dissipation relation using the same mobility tensor. The angular velocity is measured in units of $B_{\mathrm{s}} F_{\theta} / R$ and the simulation temperature is measured in units of $F_{\theta} \sigma^{2} /\left(k_{\mathrm{B}} R\right)$. In these units, the Péclet number is $\mathrm{Pe}=(R / \sigma) T^{-1}=5 / T$.

Figure 2(d) presents simulation results for two particles on a ring, confirming the role of radial symmetry breaking in creating particle pairs. At low temperature and weak radial confinement, the particles form permanent pairs. Paired particles follow trajectories of different radii with $R_{1} / R_{2} \simeq 1.01$ [Fig. 2(d) inset]. Pairing in simulation is more pronounced than in experiment, with a smaller and more narrowly distributed angular separation [Fig. 2(e) inset] 23]. For infinitely strong radial confinement, no pairing is observed. Vigorous diffusion at elevated temperatures overcomes the hydrodynamic pairing mechanism and allows pairs to move apart. This increases their drag, and reduces their mean angular velocity [Fig. 2(e)].

Simulations for rings with even $N>2$ exhibit pairing as well. Comparing the collective mobilities with our simplified model [Fig. 3(b)], we obtain a reasonable fit using the value $\alpha=1.57 \pm 0.05$. The value of $\alpha=B_{\mathrm{p}} / B_{\mathrm{s}}$ can be related also to the inter-particle distance through the theoretical expression for the mobility of a pair of spheres [18]. In the simulation we measure an angular separation of $\Delta \theta=12.9 \pm 0.1^{\circ}$, which yields $\alpha=1.52 \pm 0.02$. These two independent measurements of the pair mobility agree to within the statistical error. The smaller experimental 
value of $\alpha$ [Fig. 3(a)] suggests still weaker pairing. These quantitative differences between experiment and simulation may be related to such effects as the roughness of the experimental force landscape [16] and a possible nonintrinsic repulsion [23].

At low temperatures, particles may assemble into different configurations as a result of their tendency to form pairs, giving rise to a rich behavior of degenerate limit cycles. The simplest example is the case of three particles [10, 24]. The breaking of a triplet into a faster pair and a slower single particle exists also in the absence of curvature and radial freedom, yet, in simulations we observe long-term pairing and the consequent limit cycle of an alternating pair only when the radial confinement is made sufficiently weak.

A simulated four-particle vortex is found to converge into three stable limit cycles, shown in Fig. 4. For a given radial confinement strength, particles adopt configurations 1 and 3 at low temperatures and configurations 2 and 3 at higher temperatures. With increasing temperature the pairs gradually break. In configuration 1 the two pairs are separated by a certain angle $(0.6 \pi$ in our simulation), which seems unrelated to the system's geometry. The transition between configurations 1 and 2 explains the difference in collective mobility seen in Fig. 3(b). Since configuration 2 is compatible with our calculation of equidistant pairs, we get a better agreement for the higher temperature in which it is stabilized. This effect might also contribute to the increased mobility of four particles seen experimentally [Fig. 3(a)]. The assumed experimental Péclet numbers [16] correspond to simulation temperatures of $T=0.055$ and 0.068 for systems $\mathrm{A}$ and $\mathrm{B}$, respectively, with $\kappa$ values around 30 . This might indicate that the experiments lie in the intermediate region between pairing and unpairing (Fig. 4). As the number of particles increases so does the number of limit cycles, leading to a more complex picture to be presented elsewhere.

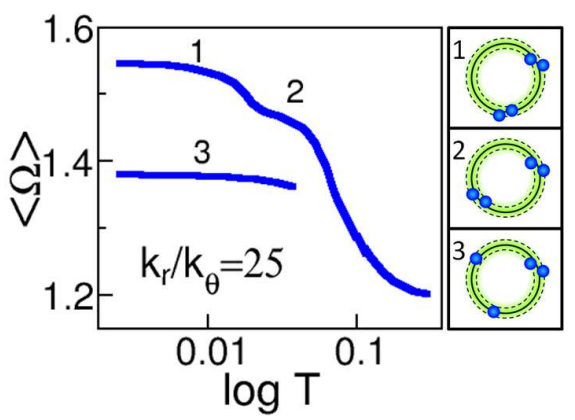

FIG. 4: Simulation results for the normalized average angular velocity $\Omega$ and particle configuration, as a function of temperature, in a four-particle vortex. Insets illustrate the three configurations.

The spontaneous symmetry breaking and dynamic coupling presented here can arise whenever particles are driven through a fluid medium along curving trajectories, provided that they are allowed to slightly move in the radial direction. Such nonequilibrium pair attractions, therefore, should affect the behavior of diverse out-ofequilibrium colloidal systems. In addition, we intend to use this model system to study further issues of nonequilibrium physics in a well controlled environment.

This study was supported by the Israel Science Foundation (Grants Numbers 1271/08, 588/06, and 8/10).

* Electronic address: roichman@tau.ac.il

[1] P. R. Nott and J. F. Brady, J. Fluid Mech. 275, 157 (1994). L. G. Leal, Phys. Fluids 16, 1833 (2004).

[2] Y.-C. Fung, Biomechanics Circulation, 2nd ed., Springer, New York, 1997.

[3] S. Ramaswamy, Adv. Phys. 50, 297 (2001).

[4] J. Dzubiella, H. Löwen, and C. N. Likos, Phys. Rev. Lett. 91, 248301 (2003).

[5] C. Mejia-Monasterio and G. Oshanin, Soft Matter 7, 993 (2011).

[6] T. M. Squires and M. P. Brenner, Phys. Rev. Lett. 85, 4976 (2000). T. M. Squires, J. Fluid Mech. 443, 403 (2001).

[7] T. Beatus, R. Bar-Ziv, and T. Tlusty, Nat. Phys. 2, 743 (2006); Phys. Rev. Lett. 99, 124502 (2007).

[8] E. R. Dufresne and D. G. Grier, Rev. Sci. Instrum. 69, 1974 (1998). J. E. Curtis, B. A. Koss, and D. G. Grier, Opt. Commun. 207, 169 (2002). J. E. Curtis and D. G. Grier, Phys. Rev. Lett. 90, 133901 (2003).

[9] M. Polin, K. Ladavac, S.-H. Lee, Y. Roichman, and D. G. Grier, Opt. Express 13, 5831 (2005).

[10] Y. Roichman, D. G. Grier, and G. M. Zaslavsky, Phys. Rev. E 75, 020401 (2007).

[11] N. B. Simpson, K. Dholakia, L. Allen, and M. J. Padgett, Opt. Lett. 22, 52 (1997).

[12] A. Ashkin, J. M. Dziedzic, J. E. Bjorkholm, and S. Chu, Opt. Lett. 11, 288 (1986).

[13] Y. Roichman, B. Sun, Y. Roichman, J. Amato-Grill, and D. G. Grier, Phys. Rev. Lett. 100, 013602 (2008).

[14] Y. Roichman, A. S. Waldron, E. Gardel, and D. G. Grier, App. Opt. 45, 3425 (2005).

[15] J. C. Crocker and D. G. Grier, J. Colloid Interface Sci. 179, 298 (1996).

[16] The issue of whether thermal fluctuations or the vortex inhomogeneities are the dominant source of noise remains unclear and requires a more detailed study.

[17] See EPAPS Document No. XXX for a movie of a twoparticle vortex and for a theoretical visualization of the instantaneous flow field created by two rotating particles.

[18] J. Happel and H. Brenner, Low Reynolds Number Hydrodynamics, Martinus Nijhoff, The Hague, 1983.

[19] J. F. Brady and G. Bossis, Ann. Rev. Fluid Mech. 20, 111 (1988).

[20] J. D. Weeks, D. Chandler, and H. C. Andersen, J. Chem. Phys. 54, 5237 (1971).

[21] J. Rotne and S. Prager, J. Chem. Phys. 50, 4831 (1969).

[22] This description is strictly valid for sufficiently large inter-particle distances. Yet, since the pairs have a finite 
separation 23], we do not expect the deviations at short distances to lead to a qualitatively different behavior.

[23] The minimum interparticle distance within a pair is about 1.7 particle diameters in the experiment and 1.1 in the simulation. We currently cannot account for the nonintrinsic repulsive effect in the experiment that might underly this difference.

[24] M. Reichert and H. Stark, J. Phys. Condens. Matter 16, S4085 (2004). 